\title{
Problem statement for practical modeling of temperature fields of gyroscopes in space navigation systems
}

\author{
$V M$ Pankratov $^{1}, A V$ Golikov $^{1}, M A$ Barulina $^{1, *}, E V$ Pankratova $^{1}$, and $M V$ Efremov $^{2}$ \\ ${ }^{1}$ Institute of precision mechanics and control problems, Russian Academy of Sciences, Saratov, \\ 410028, Russia \\ ${ }^{2}$ Research and production enterprise "Antares”, Saratov, 410600, Russia
}

\begin{abstract}
The paper describes a method of studying temperature fields in fiber-optic gyroscopes that are part of complex navigation systems, using the example fiber-optic gyroscope as part of the angular velocity measurement system BIUS-M-1, developed by "Antares" (Saratov). The work relevance is due to the fact that currently existing methods for studying external and internal thermal effects on precision devices, and the resulting three-dimensional non-stationary temperature fields may be too complicated for practical application, or require significant computation and time resource. Also, not all contemporary models take into account such parameters, as low pressure and vacuum, the importance of which for devices operating in space orbital conditions cannot be underestimated. Therefore, modeling of the thermal process in various devices should be carried out at the design stage. At the same time, such modeling should not be labor-intensive, do not require large financial investment and computing resources. In this paper, the authors formulate a research problem, develop a thermal model, and present the main relations that are the components of the mathematical model of nonstationary three-dimensional inhomogeneous temperature fields in the fiber-optic gyroscope. The resulting model can be implemented quite simply in computational algorithms and software.
\end{abstract}

\section{Introduction and problem statement}

Thermal effects are one of the most significant factors that affect the accuracy, reliability, and service life of precision sensors of inertial information of various types. In some devices, the temperature-dependent component of inaccuracy reaches up to $50 \%$. Therefore, the study and analysis of thermal processes occurring in that device is an important and actual task [111]. Besides this fact, such devices used in spacecraft can operate in a vacuum and zerogravity conditions and as studies shown, in these conditions thermal effects can have a more significant impact on the operational characteristics of the device and consequently on its performance $[1,5,12]$.

This article describes a method of studying non-stationary inhomogeneous threedimensional temperature fields in fiber-optic gyroscopes (FOG) under complex temperature

\footnotetext{
*Corresponding author: marina@barulina.ru
} 
influences which have a place in various operating modes of FOG, in particular, in space conditions. Currently, FOG is widely used in navigation systems for various purposes, often in severe operating conditions, including extreme temperature disturbances that are one of the main inaccuracy reasons. Many works [1-11] have been devoted to the study of inaccuracies in gyroscopes of this type and measures to reduce them, and still not lost their actuality.

The sources of temperature effects on navigation devices are both external effects on spacecraft (solar radiation, cosmic rays, the heat reflected from the Earth) and internal sources (heating elements, the mutual thermal influence of various sensors and systems inside spacecraft, etc.). Because of these reasons, before proceeding to study what is happening in FOG from the thermal point of view, it is necessary to determine concrete thermal effects on sensors within spacecraft and navigation measurement systems. To solve this problem, the concept of hierarchical modeling of thermal fields was developed [1,5,13]. Following this concept, the first step is to determine what temperature fields occur in a navigation device containing a FOG and located in a more complex system, for example, spacecraft. In this paper considering the FOG, which is a part of the angular velocity measurement system BIUS-M1, developed by R\&D company "Antares" (Saratov) and used in the spacecraft. Studies of thermal fields in BIUS-M-1 were carried out earlier in [5].

When modeling thermal processes in BIUS-M-1 each of three fiber-optic gyroscopes included in the device was represented as a homogeneous solid-state element with average thermophysical characteristics [5]. At the next stage of work, following the concept of hierarchical modeling of thermal fields in complex devices, a detailed study is carried out in the previously considered thermal modes of the temperature fields of a separate FOG (lower level of the hierarchy), which is a part of the navigation measuring system. Such a detailed study allows us to determine the degree of influence of external temperature disturbances on individual elements of the FOG structure, and in particular, on the fiber-optic coil, the light source, and the temperature stability of which the accuracy of the sensor depends $[1-4,6,9]$.

The purpose of this work is to describe a method for studying temperature fields in fiberoptic gyroscopes that are part of complex navigation systems in operating modes on earth and orbital conditions by computer simulation.

The object of research is the fiber-optic gyroscope FOG-39, which is part of the angular velocity measurement system BIUS-M-1, developed by R\&D company “Antares” (Saratov).

To achieve this goal, the following tasks are set:

- construction of a thermal and mathematical model of a non-stationary inhomogeneous temperature field of a fiber-optic gyroscope;

- development of software realized the constructed mathematical model based on in $\mathrm{C}++$ with the included library of models of thermal effects (LMTE).

- using the developed software, obtain and analyze comparative data on the temperature distribution in the fiber-optic gyroscope in various operating modes.

\section{Mathematical model}

The considered FOG has a modular structure and consisted of elements with a heat source or sink (thermal elementary volumes). The general view with the main elements of considered FOG and his thermal model with main thermal connections are shown in Figure 1 and Figure 2.

The most significant details and design features of gyroscope, physical processes, and all types of heat exchange in it should be taken into account in the developed thermal model. 
The mathematical model is determined by the mathematical realization of the thermal model in the form of algorithms, analytical relations, equations, and formulas, that form the basis of the elementary balances method $[1,5,13,17,19]$.

For the case of calculating the temperature field of FOG the main equation has the form:

$$
T_{i}(t+\Delta t)=\left\lfloor 1-\frac{\Delta t}{c_{i}}\left(\sum_{j=1}^{N} q_{i j}+q_{i c}\right)\right\rfloor T_{i}+\frac{\Delta t}{c_{i}}\left(\sum_{j=1}^{N} q_{i j} T_{j}+q_{i c} T_{c i}+Q_{i}\right),
$$

where: $T_{i}(t), T_{i}(t+\Delta t), c_{i}(i=1, \ldots, M)$ - the temperature of $i$-th element in the present and next moment and his heat capacity; $q_{i j}$ - thermal relation between elements $i, j(j=1, \ldots, N)$; $q_{i c}$ - thermal relation between $i$-th element and environment; $T_{c i}$ - environment temperature; $Q_{i}$ - heat source power; $M$ - element quantity; $N$ - the number of elements that have heat contact with $i$-th element; $\Delta t$ - calculation step.

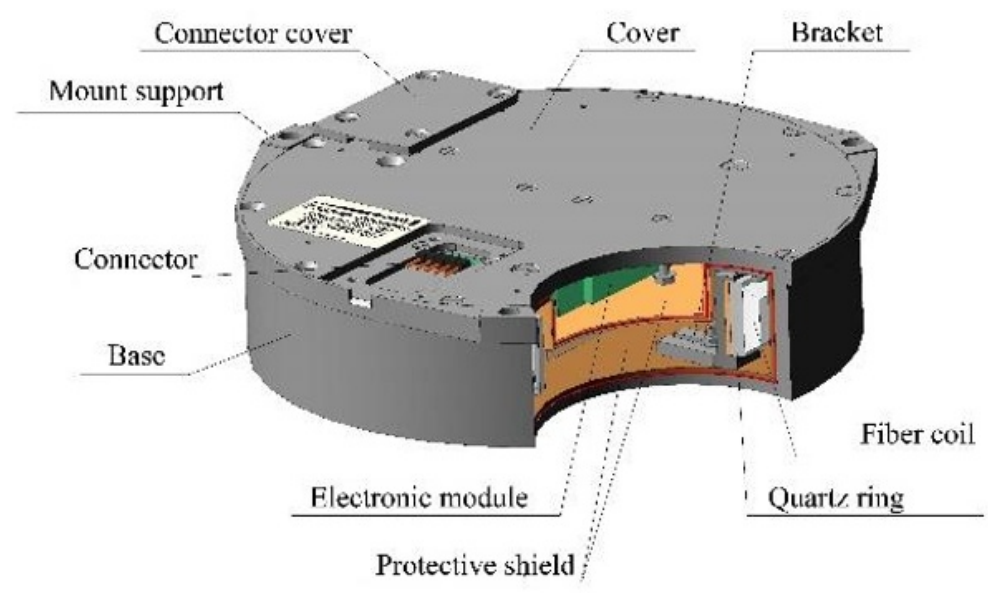

Fig. 1. General view with the main elements of FOG. 


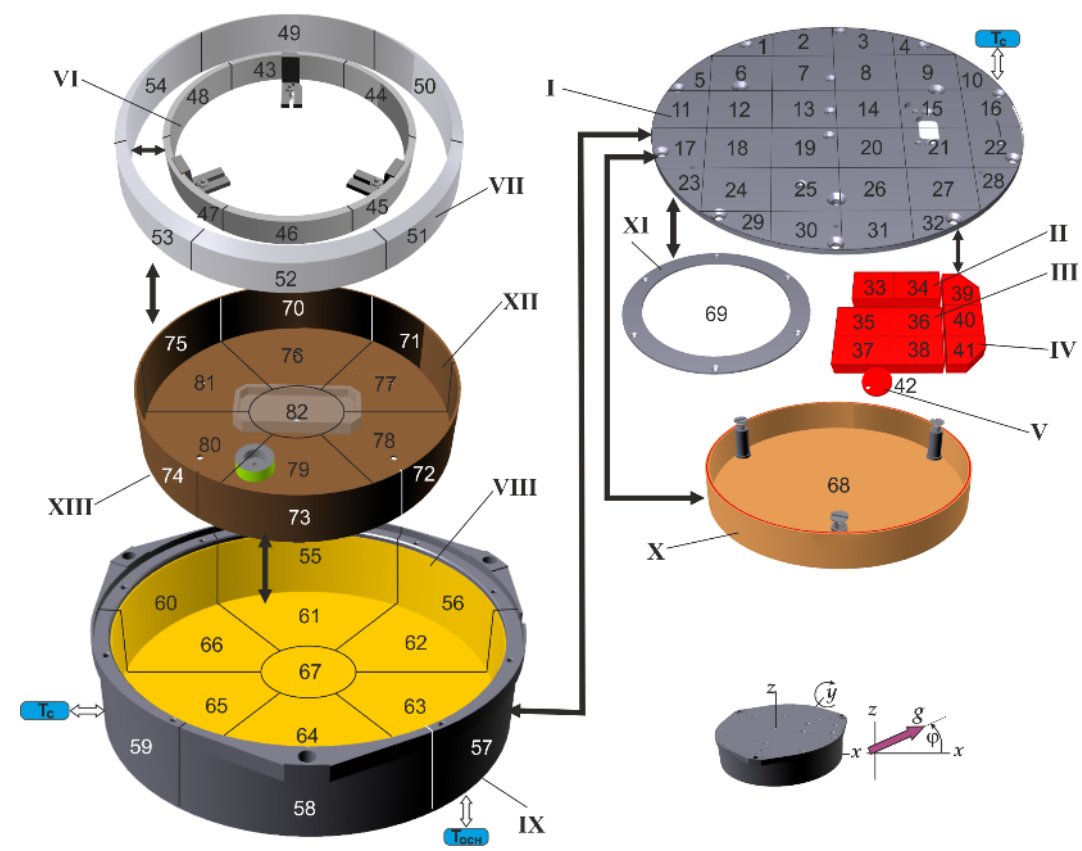

Fig. 2. Thermal model of the FOG and orientation regarding the gravity vector. Where: IXIII - layers of FOG construction, 1-82 - thermal elementary volumes.

A special feature of the developed mathematical model of the FOG is that the FOG orientation relative to the gravity vector is taken into account. It's a very important parameter because the gravity, pressure of surrounding, and interior media, the coefficients of convective heat transfer from gyroscope elements to the environment depend on gyroscope orientation in the gravity field. The basic formulas for the determination of these dependencies are determined in accordance with the free-convective heat exchange theory [17-20]. For example, the formula for calculating the coefficient of heat transfer to the environment form thermal elementary volumes with a certain orientation of gyroscopes has the following form:

$$
q_{c i}=S_{i}\left(2 \alpha_{r}+(1-0,3 \sin \varphi) \alpha_{k} g_{V} \sqrt{D_{N}}\right)
$$

$\alpha_{k}, \alpha_{r}$ - coefficients of the heat transfer by free convection and radiation;

$S_{i}$ - area of heat transfer of elementary volumes;

$D_{N}=D_{N A R} / D_{0}$ - ambient pressure $D_{N A R}$ relative to normal atmospheric pressure $D_{0}$;

$g_{V}=g / g_{0}$ - acceleration of $g$ relative to the acceleration of normal gravity $g_{0}$;

$\varphi$ - the angle of the gyroscope orientation relative to $g$.

\section{Thermal operation modes}

The stated problem was solved for different thermal modes that were defined in previous work [5]. It should be noted that the external temperature effect is exerted on the entire measuring system, and then it affects the FOGs through the system's elements with the accountant of self-disturbing thermal factors, for example, inner heat sources. Therefore, the 
data obtained during modeling of the entire measuring unit (the upper level of the hierarchy) are taken as parameters of the external temperature disturbance for the FOG (lower level of the hierarchy).

So, two thermal modes were defined earlier:

\subsection{1-st thermal mode (earthly condition)}

This mode simulates "comfortable" conditions of normal atmospheric pressure outside and inside the measurement system under study and normal gravity. All elements of the measurement system at the initial moment are considered to be warmed up to a temperature of $+20^{\circ} \mathrm{C}$, the ratio of the pressure of the environment surrounding the FOG to the normal atmospheric pressure is 1 . The ratio of the pressure inside the FOG to normal atmospheric pressure is also equal to 1 .

This thermal operation mode takes into account the heat transfer to the environment by free convection (in the presence of normal pressure and gravity) and radiation, and the conductive heat transfer from of the elements of FOG to each other.

It is simulated that all heat sources are switched on simultaneously on electronic boards and in a superluminescent diode (SLD) with a total power $\approx 0,96 \mathrm{~W}$.

A three-dimensional, inhomogeneous, non-stationary temperature field is calculated and visualized in all thermal elementary volumes ( 82 elements).

Simulation of this thermal operation mode is performed to find out the general picture of temperature field changes in the basic construction of the FOG under normal ambient pressure and normal gravity.

In this case, the maximum overheats $\Delta T_{\max }=T_{\max }-T_{c}$ of device elements under the environment are determined, and it's found out where these overheats occur, and the time of transient thermal processes is set.

In the same way, important characteristics as the maximum non-stationary and steadystate temperature circumferential $\Delta \mathrm{T}_{\psi}$ and radial $\Delta \mathrm{T}_{\mathrm{R}}$ differences in fiber-optic coil are determined.

\subsection{2-nd thermal mode (space conditions)}

This mode simulates "uncomfortable" conditions like vacuum outside and inside the measurement system and zero-gravity. It is assumed that at the initial moment all elements of the basic structure are warmed up to a temperature of $+20^{\circ} \mathrm{C}$, the pressure in the environment surrounding the system in ratio to normal atmospheric pressure is zero (vacuum), the pressure inside the unit in ratio to normal atmospheric pressure is also zero (vacuum). The following heat transfer processes are taken into account: the heat transfer to the environment by radiation, the conductive heat transfer through the FOG elements to each other, the heat transfer to the measurement system, the radiation heat transfer.

Simulations of this thermal mode are performed to determine the degree of influence of severe conditions of orbital flight on the thermal state in the FOG.

The studying the non-stationary temperature field of the FOG in the specified thermal conditions was carried for the following sets of parameters of external temperature influence: 1) The environment temperature and temperature of thermoplate, to which the FOG is attached, is constant and equal to $+40{ }^{\circ} \mathrm{C}$. 
2) The environment temperature and temperature of thermoplate, to which the FOG is attached, is changing according to a given cyclogram in the range $+40^{\circ} \mathrm{C} \div-10^{\circ} \mathrm{C}$ and imitates testing of the device in the thermal chamber.

3) The environment temperature and temperature of thermoplate, to which the FOG is attached, is changing by harmonic low with a given frequency in range $+40^{\circ} \mathrm{C} \div-10^{\circ} \mathrm{C}$.

The frequency varies within $\omega=0,00116 \div 0,0116 \mathrm{c}^{-1}$ (period $\tau=2 \pi / \omega=90 \div 9$ minutes).

This set simulates the influence of harmonically changing external temperature disturbances on thermal processes and taking into account it's heating from internal heat sources.

\section{Conclusion}

Thus, in that part of the work, the main tasks of research are formulated. The mathematical model of the FOG's temperature fields was constructed, in which the structural elements of the sensor are divided into "elementary" thermal volumes, which are the calculated points of the model.

The main relations that form the basis of a mathematical model for calculating a threedimensional non-stationary inhomogeneous temperature field of a VOG are determined.

For the thermal "elementary" volumes, the necessary thermophysical parameters (heat capacity, mass, etc.) are calculated. Thermal connections with neighboring volumes and the environment are determined. The corresponding thermal relation coefficients are calculated taking into account all types of heat exchange (conductive, convective, radiation heat exchange).

The work was supported by the RFBR grant 19-08-00839.

\section{References}

1. Djashitov V, Pankratov V 2005 Sensors, devices and systems of aerospace and marine instrumentation in the conditions of thermal effects (St.-Petersburg: SSC RF Central research Institute "Electropribor") p 404

2. Dranitsina E, Egorov D 2012 Proceedings of the XIV conference of young scientists "Navigation and traffic management» (St.-Petersburg: SSC RF Central research Institute "Electropribor") p 447

3. Dranitsina E, Untilov A, Daineka G, 2012 Gyroscopy and navigation 4 (79) 10

4. Sharkov I, Rupasov A, Volkovskii S 2013 Scientific and technical Bulletin of information technologies, mechanics and optics 6 (88) 31

5. Golikov A, Pankratov, Barulina M, Pankratova E, Efremof M 2019 Innovations and investments 11167

6. Lefevre H 1993 The Fiber-Optic Gyroscope (Norwood MA: Artech House) p 24

7. Zhihong Li, Zhuo Meng, Tiegen Liu and X Steve Yao 2013 OPTICS EXPRESS 21(2)

8. Schadt F and Mohr F 2011 Inertial Sensors and Systems - Symposium Gyro Technology (Germany: Karlsrue)

9. Shupe D M 1980 Appl. Opt. 19(5) 654-5 doi: 10.1364/AO.19.000654

10. Esipenko I, Lykov D 2017 Bulletin of Bauman Moscow state technical University. Ser. Instrument making 531 doi: 10.18698/0236-3933-2017-5-31-46 
11. Dzhashitov V E, Pankratov V M, Golikov A V, Gubanov A G and Efremov M V 2012 Gyroscopy and Navigation 3(1) 56

12. Korolev S 2006 Systems for ensuring the thermal regime of spacecraft: a textbook (St.Petersburg: Baltic state tech. university) p 100

13. Djashitov V, Pankratov V, Golikov A 2013 Gyroscopy and navigation 1(80) 49-63

14. Dzhashitov V E, Pankratov V M and Golikov A V 2008 IEEE AEROSPACE and ELECTRONIC SYSTEMS MAGAZINE 23 18-21

15. Dzhashitov V E and Pankratov V M 2009 Journal of Computer and Systems Sciences International 48(3) 481

16. Golikov A V, Pankratov V M and Efremov M V 2018 Gyroscopy and Navigation 9(2) 116

17. Dulnev G, Parfenov V, Sigalov A, 1990 Methods for calculating the thermal regime of devices (M.: Radio and communication) p 312

18. Dulnev G, Semyashkin E 1968 Heat exchange in electronic devices (L.: Energy) p 360

19. Barulina M, Golikov A, Pankratov V, Efremov M 2018 Scientific instrument engineering 28(3) 14

20. Basarab M, Kravchenko V, Matveev V 2005 Mathematical modeling of physical processes in gyroscopy (M.: Radiotechnic) p 176 